\title{
The acoustic characteristics of the Baraba-Tatar and Shor consonant ' $r$ '
}

Tatiana Ryzhikova ${ }^{1,2}$, Nikolay Urtegeshev ${ }^{1,2}$

${ }^{1}$ The Laboratory of Linguistic Anthropology, Tomsk State University, Russia

${ }^{2}$ Nadelyayev Laboratory of Experimental-Phonetic Researches, Institute of Philology, SB RAS, Russia

https://doi.org/10.36505/ExLing-2019/10/0044/000406

\begin{abstract}
The paper considers the acoustic characteristics of the Baraba-Tatar and Shor sound ' $r$ '. The experimental results show that it possesses a certain amount of noise and sometimes it is completely voiceless that makes it impossible to classify ' $r$ ' as a sonorant, but rather as a less-noise consonant. This term was proposed by V. M. Nadelyayev in 1960-s and fully describes the acoustic nature of the minority languages of Siberia and the Far East.
\end{abstract}

Key words: experimental phonetics, acoustic analysis of speech, Baraba-Tatar language, Shor language, less-noise consonants.

\section{Introduction}

The problem of endangered languages preservation, investigation and analysis is one of the most acute nowadays. Because of the globalization processes minority languages are threatened by becoming extinct. Baraba-Tatar and Shor are two languages of the indigenous population of Southern Siberia. In the UNESCO Atlas of Endangered Languages they are both listed as severely endangered (Atlas 2010) and the linguistic situation becomes worse every year.

In 1960-1980-s V. M. Nadelyayev - the founder of the Laboratory of Experimental-Phonetic Researches of the Institute of Philology of the Siberian Branch of RAS (LEPR IP SB RAS) and the outstanding investigator and the leading specialist in the phonetics of the indigenous peoples' languages of Siberia and the Far East - noticed that the generally accepted rules of classifying consonants into noise and sonorant are inappropriate and unsuitable for the languages under consideration. Thus, he suggested that the consonants usually treated as sonorant should rather be called less-noise in the Siberian and Far-Eastern languages, because they do not comprise as much noise as noise consonants, but they are also not fully sonorant. In some positions, they can be manifested as fully or partially noise and moreover voiceless (Nadelyayev 1960).

The aim of this paper is to analyze the Barabian and Shor sound ' $r$ ' and show that it should be classified not as a sonorant but rather as a less-noise consonant.

ExLing 2019: Proceedings of 10 $0^{\text {th }}$ International Conference of Experimental Linguistics, 25-27 September 2019, Lisbon, Portugal 


\section{Experiment}

The phonetic material has been collected in the places where the bearers of the two languages live: in the Baraba steppe and Mountain Shoria.

The Barabian recordings were made in 2000-2005 and 2012-2015. Presented here are the latest data. The number of speakers is 9, with their age differing from 22-25 up to 65-70 years. All of them speak Baraba-Tatar from birth, the second language is Russian, and some of them speak literary Tatar.

The experimental program included words comprising sound ' $r$ ' in all possible positions in Barabian with the Russian translation. The subjects were asked to pronounce the target words three times in their native language. The recordings were made with a digital voice recorder HandyRecorderH4n with external noise filter. Processing of the linguistic material was done in SpeechAnalyzer. The voiced and voiceless components of the sound ' $r$ ' were distinguished and their relative length calculated.

The Shor material (the Verkh-Mrass (Upper-Mrass) subdialect) was also collected in the field in 2008. The second expedition to Shoria in 2016 showed that it was impossible to collect new material because of the age limits of the subjects or because of the ethnic changes in the villages population. Thus only material of 2008 is used in this paper. The speakers are the bearers of the Shor language (the Verkh-Mrass subdialect). Their number is 9. The age group is above 72 years old. All of them speak Shor as a native language, with Russian being the second language. The program was similar to the Barabian one and the process of recording material was the same. A digital voice recorder HandyRecorderH4n with external noise filter was used. Post-processing was done in the laboratory conditions in the SpeechAnalyzer Program. Target sound is ' $r$ '.

\section{Results and discussion}

In different languages sound ' $r$ ' has different articulatory-acoustic characteristics. The common trait for all of them is the presence of a short pause surrounded by a vocal context (sometimes there are two, three or more such sequences) and it is this combination that defines a vibrant consonant (Knyazev, Pozharitskaya 2011). In Baraba-Tatar ' $r$ ' is a forelingual vibrant (Ryzhikova 2004). In Shor it is a forelingual pharyngealized vibrant (Urtegeshev 2004). Russian ' $r$ ' is a forelingual voiced vibrant (Knyazev, Pozharitskaya 2011). Figure 1 shows the examples of the Barabian, Shor and Russian sound ' $r$ ' in the position between the vowels and in the word end. 
The acoustic characteristics of the Baraba-Tatar and Shor consonant ' 11 '79

a)
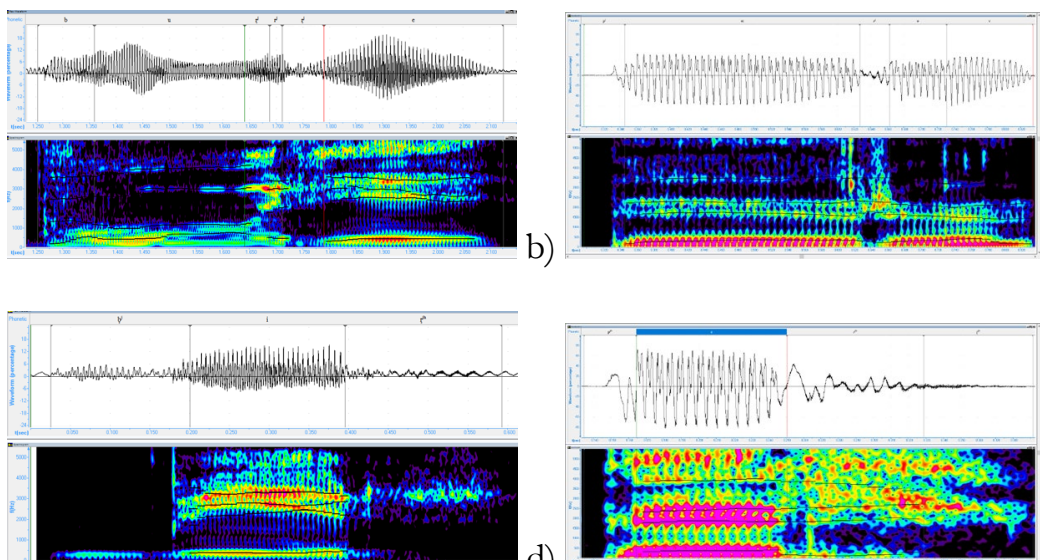

c)
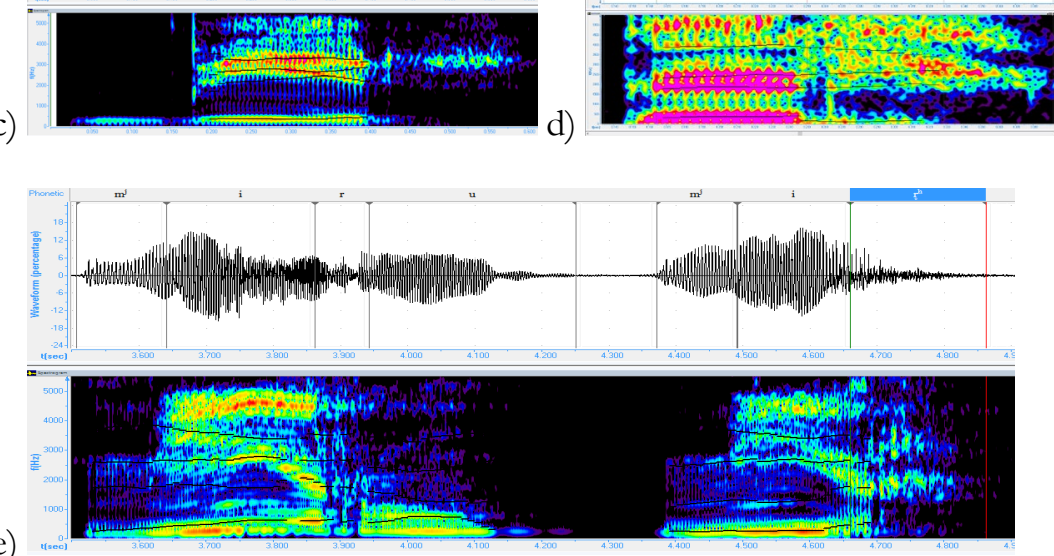

Figure 1. a) Barabian büre 'a wolf', b) Shor pörü 'a wolf, c) Barabian bir 'give!', d) Shor per 'give!', e) Russian miru mir! 'peace in the world!'

The results of the acoustic analysis of the Baraba-Tatar and Shor languages turned out to be in agreement. The 'r'-type consonant in the Verkh-Mrass subdialect of Shor can be interpreted as less-noise, because it comprises noise component in all the positions in a word and in some cases it consists only of the noise part. Nevertheless, almost all realizations of the Shor phoneme /r/ can be interpreted as voiced ones, except the position in the very end of a word. Here the presence of voice depends on the vowel harmony of a word: if it is with front vowels, the allophones of $/ \mathrm{r} /$ are voiceless (the average amount of voice is $19-57 \%$ of the sound length); if the word contains back vowels, voice comprises about $50-81 \%$.

In Baraba-Tatar, realizations of the phoneme /r/ also contain the noisy part, but the presence of the voice in their production does not seem to play an important role: even in the strongest position between two vowels the allophones of $/ \mathrm{r} /$ may be both voiced and voiceless. In the final position in the word the length of the realizations increases in 1,5 times of the average length of the sound and the number of voiceless components varies from 92 up to $100 \%$. In general, the number of voiceless components in all realizations of the 
phoneme $/ \mathrm{r} /$ is $71,3 \%$. To sum up, it can be defined as less-noise with a different degree of voiced components.

In the Russian example, one can see a considerable amount of noisy components in the very end of a word and the absence of voice, though the bearers of the Russian language do not perceive it (Knyazev, Pozharitskaya 2011).

\section{Conclusions}

To summarize, the investigation has proven that the classification of consonants at least in two Siberian Turkic languages into noise and less-noise units seems to be reasonable. Moreover, the presence and quantity of voice in the production of the phoneme $/ \mathrm{r} /$ in the languages under consideration vary significantly: in some positions the presence of voice is negligible in both of them. This fact allows us to assume that voice is not a constitutive-differential characteristic of the phoneme $/ \mathrm{r} /$ and it should be classified not as sonorant but rather as less-noise with the additional characteristic of being voiced or voiceless.

\section{Acknowledgements}

The work was financially supported by the RF Government Grant № 14.Y26.31.0014 «Linguistic and ethno-cultural diversity of Southern Siberia in synchronic and diachronic aspects: interaction of languages and cultures».

\section{References}

Knyazev, S. V., Pozharitskaya, S. K., 2011. Sovremennyi russkiy literaturnyi yazyk: Phonetika, orfoepiya, grafika i orfografiya. Moscow, Akademicheskiy proekt, Gaudeamus. (Contemporary Russian literary language: Phonetics, orthoepy, graphics and spelling).

Nadelyayev, V. M., 1960. Proyekt universal'noy unifitsirovannoy phoneticheskoy transkroptsii (UUPhT). Moscow-Leningrad. (The Project of the universal unified phonetic transcription).

Ryzhikova, T. R., Urtegeshev, N. S., 2004. Less-noise forelingual consonant phonemes in Baraba Tartars' and Shor languages. In Proc. 12th International Conference on Turkish Linguistics, Izmir, North Cyprus.

UNESCO Atlas of the World's Languages in Danger (http://www.unesco.org/languages-atlas/ last consulted 27.07.2019).

Urtegeshev, N. S., 2006. Maloshumnye soglasnye shorskogo yazyka: artikulyatsionnoakusticheskiye osobennosti (mrasskiy dialect) in Alchikova, O. et al., Tyurkskiye yazyki: problemy i issledovaniya, 77-82, Gorno-Altaisk, Russia. (Less-noise consonants of the Shor language: articulatory-acoustic peculiarities (the Mrass dialect)). 